\title{
From elliptic iterated integrals to elliptic multiple zeta values
}

\author{
Johannes Broedel* \\ Humboldt University Berlin \\ E-mail: jbroedel@physik.hu-berlin.de
}

While transcendental numbers are omnipresent in modern field-theory amplitude calculations, it is scattering amplitudes in string theory, which provide the most straightfoward setting for bridging the gap between conventional quantum field theory and number theory. In these proceedings, the relation between tree-level (genus zero) open-string integrals, harmonic polylogarithms and multiple zeta values will be reviewed. Starting from this setup, an analogy to genus-one will be drawn in order to demonstrate, how elliptic multiple zeta values appear naturally as results of planar open-string cylinder integrals.

Loops and Legs in Quantum Field Theory

24-29 April 2016

Leipzig, Germany

${ }^{*}$ Speaker. 


\section{Introduction}

While different flavors of polylogarithms [1, 2, 3] have been the backbone of numerous calculations of scattering amplitudes in quantum field theories for decades, elliptic integrals made an appearance in the computation of several quantities more recently $[4,5,6,7,8]$. Therefore it is of immediate importance to understand their structure and to investigate the transcendental numbers those integrals yield if evaluated at particular arguments.

In these proceedings, the reader shall be acquainted with a natural setting which straightforwardly leads to elliptic functions and simultaneously provides an elliptic generalization of multiple zeta values: scattering amplitudes in open string theory.

Being a conformal field theory, the determination of open-string scattering amplitudes amounts to the calculation of a correlator between string-vertex operators inserted at the boundary of Riemann surface of appropriate genus. In open string theory, each particle carries color charge, which is - as in conventional quantum field theory - implemented by a color-trace for each color-ordered amplitude. Naturally, this leads to a particular ordering of vertex operators on the boundary of the Riemann surface.

As will be explained below, the language used for the evaluation of open-string scattering amplitudes is determined by the topology of the surface in question. Even more, for each given topology contributing to a certain amplitude a particular class of iterated integrals with corresponding periods can be identified. Once this correlation is understood, powerful algebraic tools immanent in the appropriate class of iterated integrals may be employed in order to yield the desired results.

Using the example of tree-level and one-loop scattering amplitudes in open string theory, properties of the respective languages will be discussed, parallelis will be drawn and distinctions are going to be pointed out. These proceedings set out to invite the reader to consider string theory amplitudes as a laboratory for better understanding elliptic (and possibly more general) objects appearing at higher loop orders or for massive integrals in quantum field theory.

\section{Open-string tree-level amplitudes}

Open-string amplitudes at tree-level are obtained from correlators on a genus-zero Riemann surface, that is, insertions of string vertex operators carrying the external momentum $k_{i}$ on the boundary of a disk. At tree-level, open string amplitudes split [9] into a low-energy part and an associated string correction $F^{\sigma}$

$$
A_{\text {string }}^{\text {open }}\left(1,2, \ldots, n ; \alpha^{\prime}\right)=\sum_{\sigma \in S_{N-3}} F^{\sigma}\left(s_{i j}\right) A_{\mathrm{YM}}(1, \sigma(2,3, \ldots, N-2), N-1, N),
$$

where $A_{\mathrm{YM}}$ is the corresponding amplitude in the Yang-Mills theory obtained from taking the inverse string tension $\alpha^{\prime}$ to zero. The sum extends over a basis set of Yang-Mills amplitudes labeled by permutations $\sigma \in S_{N-3}$. All state dependence is carried by the field theory amplitudes $A_{\mathrm{YM}}$, which simultaneously provide the pole structure of the open-string amplitude. The string correction $F^{\sigma}$, on the other hand, is a purely kinematic correction which is a polynomial in dimensionless Mandelstam variables $s_{i_{1} \ldots i_{n}}=\alpha^{\prime}\left(k_{i_{1}}+\ldots+k_{i_{n}}\right)^{2}$ whose coefficients are rational combinations of multiple zeta values (cf. eq. (2.5) below). 
Conformal symmetry of the tree-level worldsheet allows to map the boundary of the disc to a segment of the real line as shown in figure 1. Furthermore, one has to take care for the $S L(2)$ -
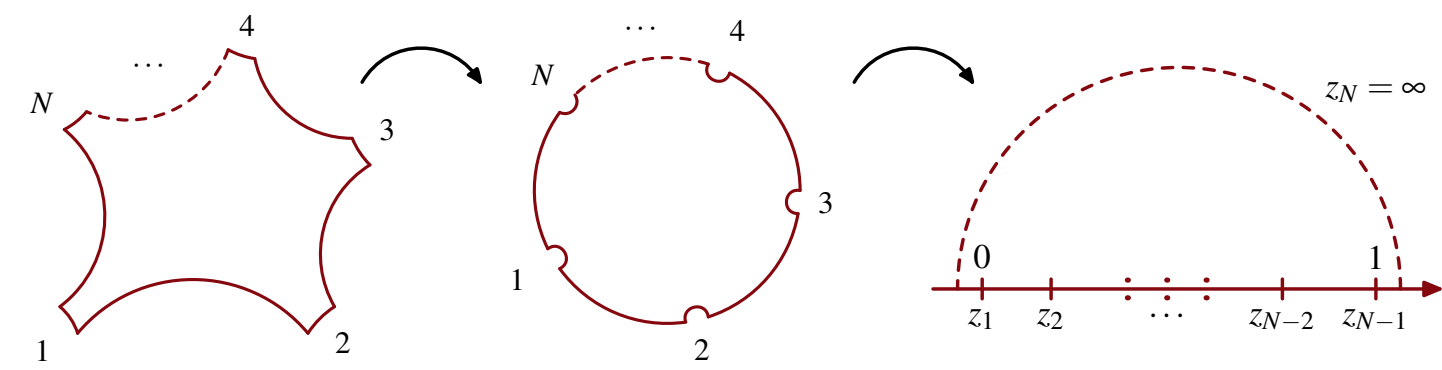

Figure 1: Employing conformal symmetry, the genus-zero worldsheet can be mapped to a configuration where the vertex insertion points $z_{1}, \ldots z_{N-1}$ are located in the interval $[0,1]$ on the real line.

symmetry of the worldsheet by fixing three insertion points to $z_{1}=0, z_{N-1}=1$ and $z_{N}=\infty$. The integral corresponding ${ }^{1}$ to the last picture in figure 1 reads:

$$
F^{2, \ldots, N-2}=\prod_{i=2}^{N-2} \int_{0}^{z_{i+1}} \frac{\mathrm{d} z_{i}}{z_{i}-a_{i}} \prod_{i<j}^{N-1}\left|z_{i j}\right|^{s_{i j}} \stackrel{\text { reg. }}{\Rightarrow} \prod_{i=2}^{N-2} \int_{0}^{z_{i+1}} \frac{\mathrm{d} z_{i}}{z_{i}-a_{i}} \prod_{i<j}^{N-1} \sum_{n_{i j}=0}^{\infty}\left(s_{i j}\right)^{n_{i j}} \frac{\left(\ln \left|z_{i j}\right|\right)^{n_{i j}}}{n_{i j} !}
$$

where the variables $a_{i}$ in the above equation are taken from the set of the other insertion points $z_{j \neq i}$. The term $\left|z_{i j}\right|^{s_{i j}}$ is the so-called Koba-Nielsen-factor: it is the propagator on the genuszero worldsheet and leads - upon expansion - to logarithms of the difference between insertion points $z_{i j}=z_{i}-z_{j}$. Clearly, the first form of the integral in eq. (2.2) is divergent whenever two neighboring vertex positions agree. Those divergences have to be regulated and their poles turn out to be expressible in terms of lower-point string amplitudes [10]. As indicated in eq. (2.2), here we will consider integrals which have already been regulated.

Obviously, one is dealing with iterated integrals, which - due to the color-ordering of the external legs - are equipped with a natural ordering. Considering the differential $\frac{\mathrm{d} t}{t-a_{i}}$ and in addition the appearance of the logarithm in eq. (2.2), it is straightforward to identify Goncharov polylogarithms as an appropriate language for this type of iterated integrals. Goncharov polylogarithms [3] with label $\vec{a}$ and argument $z$ are defined recursively via

$$
G\left(a_{1}, a_{2}, \ldots, a_{n} ; z\right)=\int_{0}^{z} \frac{\mathrm{d} t}{t-a_{1}} G\left(a_{2}, \ldots, a_{n} ; t\right), \quad G(; z)=1, \quad G(\vec{a} ; 0)=G(; 0)=0
$$

and are related to usual logarithms by

$$
G(\underbrace{0,0, \ldots, 0}_{w} ; z)=\frac{1}{w !}(\ln z)^{w} \quad G(\underbrace{1,1 \ldots, 1}_{w} ; z)=\frac{1}{w !} \ln ^{w}(1-z) .
$$

If evaluated at $z=1$, Goncharov polylogarithms with $a_{i} \in\{0,1\}$ reduce to multiple zeta values

$$
\zeta\left(n_{1}, \ldots, n_{r}\right)=(-1)^{r} G(\underbrace{0,0, \ldots, 0,1}_{n_{r}}, \ldots, \underbrace{0,0, \ldots, 0,1}_{n_{1}} ; 1) .
$$

\footnotetext{
${ }^{1}$ For the sake of simplicity, only the expression for the trivial permutation will be noted here. Results for all other permutations can be obtained straightforwardly using the methods described in refs. [9, 10].
} 
which need to be shuffle-regularized [11]. Upon expressing integrals of the type (2.2) in terms of Goncharov polylogarithms, the evaluation of the result amounts to employing applications of their rich algebraic structure. In particular does the straightforward rewriting of the integral (2.2) lead to polylogarithms where $a_{i} \in\left\{0,1, z_{i}\right\}$. However, as explained and elaborated on in ref. [10], all polylogarithms of this particular kind can be expressed as linear combinations of polylogarithms with labels from $\{0,1\}$ exclusively, which in turn can be cast into multiple zeta values using eq. (2.5). As an example, the string correction to the five-point amplitude ${ }^{2}$ for the trivial permutation reads

$$
\begin{aligned}
F^{23}= & 1-\zeta(2)\left(s_{12} s_{23}+s_{12} s_{24}+s_{12} s_{34}+s_{13} s_{34}+s_{23} s_{34}\right) \\
& +\zeta(3)\left(s_{12}^{2} s_{23}+s_{12} s_{23}^{2}+s_{12}^{2} s_{24}+2 s_{12} s_{23} s_{24}+s_{12} s_{24}^{2}+\cdots\right)+\cdots \\
& +\zeta(3,5)(\ldots)+\cdots
\end{aligned}
$$

where one easily recognizes the pattern described after eq. (2.2). Furthermore, $F^{\sigma}$ is of uniform transcendentality: the power of Mandelstam variables matches the transcendentality of the accompanying zeta value. Of course, in the limit $\alpha^{\prime} \rightarrow 0$ of eq. (2.1) one needs to recover the field theory amplitude: this is implemented by $F^{2 \ldots N-2} \rightarrow 1$ and all other $F^{\sigma}$ vanish for vanishing $\alpha^{\prime}$.

A crucial concept in massaging the integrals in eq. (2.2) is the use of partial fraction identities

$$
\int_{0}^{z} \mathrm{~d} w \frac{1}{w-a_{1}} \frac{1}{w-a_{2}}=\int_{0}^{z} \mathrm{~d} w\left(\frac{1}{\left(w-a_{1}\right)\left(a_{1}-a_{2}\right)}+\frac{1}{\left(w-a_{2}\right)\left(a_{2}-a_{1}\right)}\right)
$$

in order to bring integrals in a form where definition eq. (2.3) can be applied. While mentioning this fact might appear unnecessary here, one will see that partial fraction is replaced by a more sophisticated set of identities for the one-loop setup discussed in the next section.

Although the formalism described above is capable to even deliver results in an automatized way, the setting of open-string tree amplitudes combined with the power of the Hopf algebraic structure governing (motivic) multiple zeta values $[14,15,16]$ allows to formulate the problem in a way which finally delivers the (properly regulated) result without the need of calculating any integrals at all: one can obtain the complete $\alpha^{\prime}$-expansion of tree-level open-string amplitudes from a suitable representation of the Drinfeld associator $[17,18]$ acting on a vector $\hat{F}\left(z_{0}\right)$, which contains all integrals of type (2.2) augmented by an auxiliary insertion point $z_{0}$. The idea is as follows [19, 20]: for the Knizhnik-Zamolodchikov equation

$$
\frac{\mathrm{d} \hat{F}\left(z_{0}\right)}{\mathrm{d} z_{0}}=\left(\frac{e_{0}}{z_{0}}-\frac{e_{1}}{z_{0}-1}\right) \hat{F}\left(z_{0}\right)
$$

the Drinfeld associator $\Phi$, which can be written [21] as a generating series of multiple zeta values ${ }^{3}$

$$
\Phi\left(e_{0}, e_{1}\right)=\sum_{w \in\{0,1\} \times} e_{w_{1}} e_{w_{2}} e_{w_{3}} \cdots \zeta\left(\cdots w_{3} w_{2} w_{1}\right)=1+\zeta(2)\left[e_{0}, e_{1}\right]+\zeta(3)\left[e_{0}-e_{1},\left[e_{0}, e_{1}\right]\right]+\cdots
$$

\footnotetext{
${ }^{2}$ Initially, this calculation has been addressed via hypergeometric functions in refs. $[12,13]$ and using the pure spinor superspace in ref. [9].

${ }^{3}$ The sum extends over all words $w=w_{1} w_{2} w_{3} \ldots$ with letters taken from the alphabet $\{0,1\}$.
} 
acts as the monodromy connecting the regularized boundary values at the two singular points

$$
C_{0} \equiv \lim _{z_{0} \rightarrow 0} z_{0}^{-e_{0}} \hat{F}\left(z_{0}\right), \quad C_{1} \equiv \lim _{z_{0} \rightarrow 1}\left(1-z_{0}\right)^{e_{1}} \hat{F}\left(z_{0}\right)
$$

via

$$
C_{1}=\Phi\left(e_{0}, e_{1}\right) C_{0}
$$

In the above equations, $e_{0}$ and $e_{1}$ are generators of a free Lie algebra, whose representation in terms of Mandelstam variables is linear and which can be constructed explicitly. One can understand the auxiliary parameter $z_{0}$ as interpolating between the $(N-1)$-point and the $N$-point amplitude: located between $z_{N-2}$ and $z_{N-1}$, it will squeeze all insertion points $z_{2} \ldots z_{N-2}$ to a tiny interval close to $z_{1}=0$ in the limit $z_{0} \rightarrow 0$. From this perspective, the point $z_{N-1}$ appears to be infinitely far away in terms of the size of the tiny interval and thus does effectively take a similar rôle as $z_{N}=\infty$. Thus, one will recover the $N-1$-point amplitude.
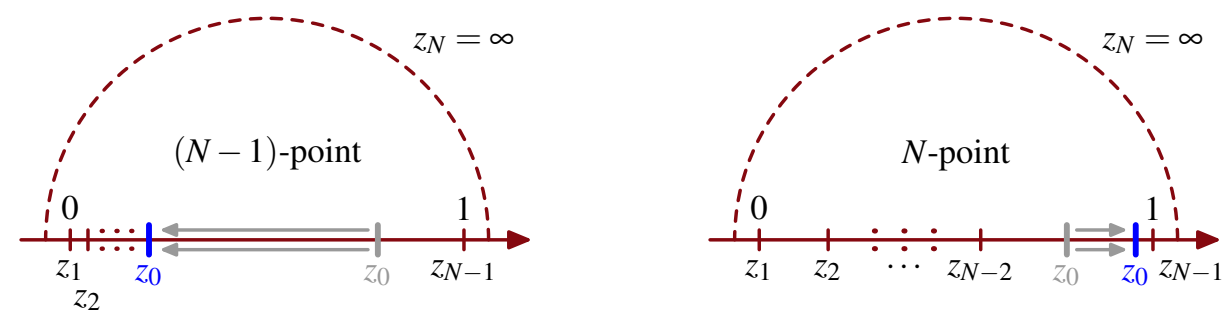

A similar argument explaining the limit $z_{0} \rightarrow 1$ together with a precise mathematical construction of the representations of $e_{o}, e_{1}$ and $\hat{F}\left(z_{0}\right)$ can be found in ref. [20].

The above calculations rely on a multitude of additional techniques and concepts, one of which shall be mentioned here explicitly: in order to compare different expressions, a canonical representation of multiple zeta values is necessary. A suitable representation which incorporates all stuffle relations has been constructed in ref. [14]. In practice, one will either make use of this basis as exemplified in ref. [22] or alternatively employ the multiple zeta value datamine [23] in order to rewrite zeta values in an empirical basis.

\section{Open string one-loop amplitudes}

Progressing to one-loop scattering amplitudes in open string theory, one has to consider a conformal field theory correlator on a Riemann surface of genus one. There are three relevant topologies for the one-loop open-string amplitude: the "planar cylinder", which is conformally equivalent to a disc with insertion points on one boundary only, the "nonplanar cylinder" which exhibits insertion points on both boundaries and the Möbius strip. For the current proceedings, only the planar cylinder, which is depicted in figure 2 will be considered. In a similar manner as in the tree-level setup, one can employ conformal symmetry in order to map the planar cylinder worldsheet into a form more suitable for calculations: for open-string one-loop amplitudes this is going to be an elliptic curve. The splitting into a field-theory amplitude and a string correction as encountered at tree-level in eq. (2.1) does not occur for one-loop amplitudes with more than five 

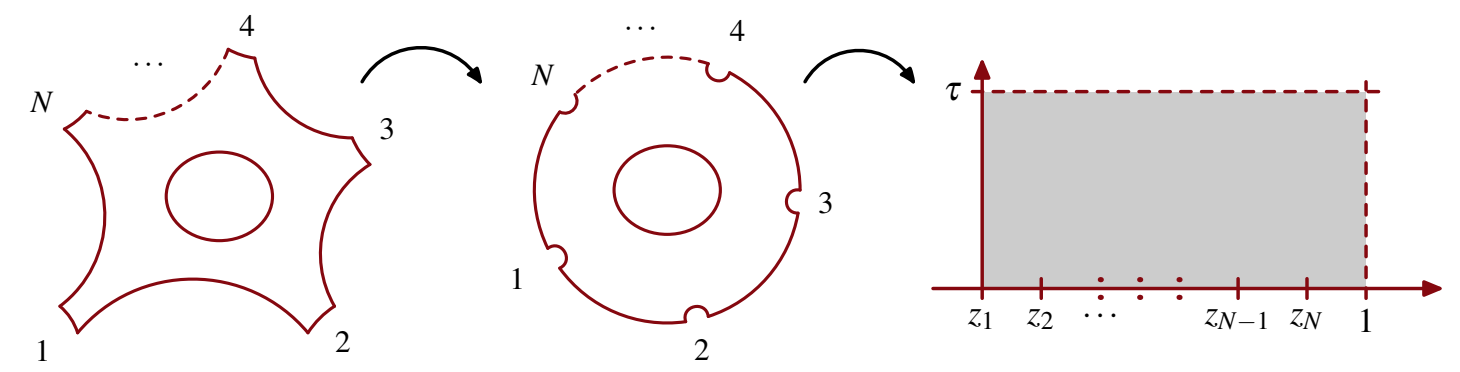

Figure 2: Using conformal symmetry, the genus-one worldsheet can be mapped to an elliptic curve. The vertex insertion points $z_{1}, \ldots z_{N}$ are chosen to be located in the interval $[0,1]$ on the real line.

external particles. Therefore a four-point example will be used here, where this feature persists. The relevant four-point integral for this topology reads [24]

$$
\begin{aligned}
A_{\text {string }}^{1-\text { loop }}(1,2,3,4) & =s_{12} s_{23} A_{\mathrm{YM}}^{\text {tree }}(1,2,3,4) \int_{0}^{\infty} \mathrm{d} \tau I_{4 \mathrm{pt}}(1,2,3,4)(\tau) \\
I_{4 \mathrm{pt}}(1,2,3,4)(\tau) & =\int_{0}^{1} \mathrm{~d} z_{4} \int_{0}^{z_{4}} \mathrm{~d} z_{3} \int_{0}^{z_{3}} \mathrm{~d} z_{2} \prod_{i<j}^{4} \exp \left[s_{i j} P_{i j}(\tau)\right] \\
& =\int_{0}^{1} \mathrm{~d} z_{4} \int_{0}^{z_{4}} \mathrm{~d} z_{3} \int_{0}^{z_{3}} \mathrm{~d} z_{2} \prod_{i<j}^{4} \sum_{n_{i j}=0}^{\infty} \frac{1}{n_{i j} !}\left(s_{i j}\right)^{n_{i j}}\left[P_{i j}(\tau)\right]^{n_{i j}},
\end{aligned}
$$

where the one-loop Koba-Nielsen factor again has been expanded in the last line. Note that, contrary to the situation at tree-level, one needs to fix the position of a single vertex operator only because of the symmetries of the elliptic curve. The analogy between tree-level amplitudes and one-loop amplitudes can be understood from the following correspondence ${ }^{4}$

$$
\ln \left|z_{i j}\right|=\int_{z_{j}}^{z_{i}} \frac{\mathrm{d} t}{t-z_{j}} \quad \Leftrightarrow \quad P_{i j}(\tau)=\int_{z_{j}}^{z_{i}} \mathrm{~d} w f^{(1)}\left(w-z_{j}, \tau\right) .
$$

The one-loop propagator $P_{i j}(\tau)$ can be written [25] as an integral over the function $f^{(1)}\left(w-a_{1}\right)$, which is a doubly-periodic function

$$
f^{(n)}(z, \tau)=f^{(n)}(z+1, \tau) \quad \text { and } \quad f^{(n)}(z, \tau)=f^{(n)}(z+\tau, \tau)
$$

arising as the first nontrivial expansion coefficient of the proper doubly-periodic completion of the Kronecker-Eisenstein series $[26,28] F(z, \alpha, \tau) \equiv \frac{\theta_{1}^{\prime}(0, \tau) \theta_{1}(z+\alpha, \tau)}{\theta_{1}(z, \tau) \theta_{1}(\alpha, \tau)}$ :

$$
\alpha \exp \left(2 \pi i \alpha \frac{\mathfrak{I}(z)}{\mathfrak{I}(\tau)}\right) F(z, \alpha, \tau)=\sum_{n=0}^{\infty} f^{(n)}(z, \tau) \alpha^{n}
$$

In other words, the integral weight of the form $\frac{\mathrm{d} t}{t-a_{i}}$ at tree-level will be replaced by an infinite number of integration weights $f^{(n)}$ in the one-loop setup. Therefore, in perfect analogy to eq. (2.3), one can define elliptic iterated integrals (or eIIs for short) [25]

$$
\Gamma\left(\begin{array}{cccc}
n_{1} & n_{2} & \ldots & n_{r} \\
a_{1} & a_{2} & \ldots & a_{r}
\end{array} ; z\right) \equiv \int_{0}^{z} \mathrm{~d} w f^{\left(n_{1}\right)}\left(w-a_{1}\right) \Gamma\left(\begin{array}{cccc}
n_{2} & \ldots & n_{r} \\
a_{2} & \ldots & a_{r}
\end{array} ; w\right)
$$

\footnotetext{
${ }^{4}$ Note that in writing down the first part of eq. (3.2) one has to make use of a regularization prescription allowing seamless conversions between Goncharov polylogarithms and usual logarithms.
} 
which now carry two labels for each iteration: the label $n_{i}$ for the integration weight and the label $a_{i}$ for the shift. These eIIs are closely related to the multiple elliptic polylogarithms considered in ref. [27, 28] but lack, however, some of their properties. Considering eIIs for integer values of $a_{i}$ (which because of eq. (3.3) is equivalent to setting $a_{i}=0 \forall i$ ), one defines elliptic multiple zeta values (or eMZVs for short) [29] via

$$
\begin{aligned}
\omega\left(n_{1}, n_{2}, \ldots, n_{r}, \tau\right) & \equiv \int_{0 \leq z_{i} \leq z_{i+1} \leq 1} f^{\left(n_{1}\right)}\left(z_{1}, \tau\right) \mathrm{d} z_{1} f^{\left(n_{2}\right)}\left(z_{2}, \tau\right) \mathrm{d} z_{2} \ldots f^{\left(n_{r}\right)}\left(z_{r}, \tau\right) \mathrm{d} z_{r} \\
& =\Gamma\left(n_{r}, \ldots, n_{2}, n_{1} ; 1\right)=\Gamma\left(\begin{array}{cccc}
n_{r} & \begin{array}{c}
n_{r-1} \\
0
\end{array} & \ldots & n_{1} \\
0 & \ldots & \ldots & 0
\end{array}\right] .
\end{aligned}
$$

Using this language, all integrals appearing in the expansion eq. (3.1) can be evaluated. In order to, however, express all integrals from eq. (3.1) in terms of eMZVs, at intermediate steps one needs to employ a generalization of partial fraction eq. (2.7) applicable to the integration weights $f^{(n)}$. This generalization comes in the form of the Fay trisecant equation [30, 31], which relates Eisenstein-Kronecker series of different arguments:

$$
F\left(z_{1}, \alpha_{1}\right) F\left(z_{2}, \alpha_{2}\right)=F\left(z_{1}, \alpha_{1}+\alpha_{2}\right) F\left(z_{2}-z_{1}, \alpha_{2}\right)+F\left(z_{2}, \alpha_{1}+\alpha_{2}\right) F\left(z_{1}-z_{2}, \alpha_{1}\right) .
$$

Upon expansion, one finds the analogue of eq. (2.7) for the elliptic setup

$$
\begin{aligned}
\int_{0}^{z} \mathrm{~d} w f^{1}\left(w-a_{1}\right) f^{1}(w)=\int_{0}^{z} \mathrm{~d} w\left(f^{(1)}\left(w-a_{1}\right) f^{(1)}\left(a_{1}\right)-f^{(1)}(w) f^{(1)}\left(a_{1}\right)\right. \\
\left.+f^{(2)}(w)+f^{(2)}\left(a_{1}\right)+f^{(2)}\left(w-a_{1}\right)\right) .
\end{aligned}
$$

Using the formalism described above, the result for the integral eq. (3.1) up to order $\alpha^{\prime 3}$ reads [25]:

$$
\begin{aligned}
I_{1234}\left(\tau, s_{i j}\right)= & \omega(0,0,0)-2 \omega(0,1,0,0)\left(s_{12}+s_{23}\right)+2 \omega(0,1,1,0,0)\left(s_{12}^{2}+s_{23}^{2}\right) \\
& -2 \omega(0,1,0,1,0) s_{12} s_{23}+\beta_{5}\left(s_{12}^{3}+2 s_{12} s_{23}\left(s_{12}+s_{23}\right)+s_{23}^{3}\right) \\
& +\beta_{2,3} s_{12} s_{23}\left(s_{12}+s_{23}\right)+\mathscr{O}\left(\alpha^{\prime 4}\right)
\end{aligned}
$$

where

$$
\begin{aligned}
\beta_{5}= & \frac{4}{3}\left[\omega(0,0,1,0,0,2)+\omega(0,1,1,0,1,0)-\omega(2,0,1,0,0,0)-\zeta_{2} \omega(0,1,0,0)\right] \\
\beta_{2,3}= & \frac{1}{3} \omega(0,0,1,0,2,0)-\frac{3}{2} \omega(0,1,0,0,0,2)-\frac{1}{2} \omega(0,1,1,1,0,0) \\
& -2 \omega(2,0,1,0,0,0)-\frac{4}{3} \omega(0,0,1,0,0,2)-\frac{10}{3} \zeta_{2} \omega(0,1,0,0) .
\end{aligned}
$$

While the above setup is very promising, it does straightforwardly lead to the question for a canonical representation of eMZVs. Fortunately, this representation is available; the keywords associated to this technique are iterated Eisenstein integrals and a special derivation algebra and the reader is hereby referred to ref. [32] and references therein for an elaborate discussion of the technique and the relation to current questions in number theory. Accompanying ref. [32], an online repository is available in which relations between eMZVs are listed to high length and 
transcendentality [33]. Furthermore, given the availability of the Drinfeld construction for openstring tree-level amplitudes, it is natural to ask for the existence of a similar construction for the elliptic setup. While a suitable elliptic generalization of the Drinfeld associator does indeed exist $[34,35,36]$, it is not yet clear, how to tackle the construction of suitable representations leading to a purely algebraic formalism delivering all open-string scattering amplitudes at the one-loop level.

\section{Conclusion}

The language of Goncharov polylogarithms which allowed to formalize the calculation of open-string tree-level scattering amplitudes can be lifted to a language suitable for the description of one-loop open-string amplitudes with vertex insertions on a single boundary. While seemingly more involved on first inspection, the main players, elliptic multiple zeta values, turn out to be beautiful objects being governed by algebraic properties very similar to those of their tree-level cousins, multiple zeta values. Further questions beyond the existence of a Drinfeld-like construction for one-loop amplitudes concern the existence of single-valued elliptic multiple zeta values as well as a further lifting of the setup to open-string scattering amplitudes at genera higher than one.

\section{References}

[1] E. Remiddi and J. A. M. Vermaseren, Int. J. Mod. Phys. A 15, 725 (2000) [hep-ph/9905237].

[2] T. Gehrmann and E. Remiddi, Nucl. Phys. B 601, 287 (2001) [hep-ph/0101124].

[3] A.B. Goncharov, Advances in Mathematics, 241, 79-102 (2013) [arXiv:0908.2238].

[4] S. Caron-Huot and K. J. Larsen, JHEP 1210, 026 (2012) [arXiv:1205.0801].

[5] L. Adams, C. Bogner and S. Weinzierl, J. Math. Phys. 54, 052303 (2013) [arXiv:1302.7004].

[6] S. Bloch and P. Vanhove, J. Number Theor. 148, 328 (2015) [arXiv:1309.5865].

[7] L. Adams, C. Bogner and S. Weinzierl, J. Math. Phys. 55, no. 10, 102301 (2014) [arXiv:1405.5640].

[8] J. M. Henn, J. Phys. A 48, 153001 (2015) [arXiv:1412.2296].

[9] C. R. Mafra, O. Schlotterer and S. Stieberger, Nucl. Phys. B 873, 419 (2013) [arXiv:1106.2645].

[10] J. Broedel, O. Schlotterer and S. Stieberger, Fortsch. Phys. 61, 812 (2013) [arXiv:1304.7267].

[11] G. Racinet, Publ. Math. Inst. Hautes Etudes Sci., 185 (2002).

[12] D. Oprisa and S. Stieberger, [hep-th/0509042].

[13] S. Stieberger and T. R. Taylor, Phys. Rev. D 74 (2006) 126007 [hep-th/0609175].

[14] F. Brown, Ann. Math. 175 (2012) 949-976.

[15] C. Duhr, JHEP 1208, 043 (2012) [arXiv:1203.0454].

[16] F. Brown, [arXiv:1102.1310], in 'Galois-Teichmüller theory and Arithmetic Geometry', Advanced Studies in Pure Mathematics.

[17] V. G. Drinfeld, Leningrad Math. J 1 (1989) 1419.

[18] V. G. Drinfeld, Leningrad Math. J 2 (1991), no. 4, 829. 
[19] J. M. Drummond and E. Ragoucy, JHEP 1308, 135 (2013) [arXiv:1301.0794].

[20] J. Broedel, O. Schlotterer, S. Stieberger and T. Terasoma, Phys. Rev. D 89, no. 6, 066014 (2014) [arXiv:1304.7304].

[21] T. Le and J. Murakami, Nagoya Math J. 142, 93 (1996).

[22] O. Schlotterer and S. Stieberger, J. Phys. A 46 (2013) 475401 [arXiv:1205.1516].

[23] J. Blumlein, D. J. Broadhurst and J. A. M. Vermaseren, Comput. Phys. Commun. 181, 582 (2010) [arXiv:0907.2557].

[24] M. B. Green, J. H. Schwarz and L. Brink, Nucl. Phys. B 198 (1982) 474.

[25] J. Broedel, C. R. Mafra, N. Matthes and O. Schlotterer, JHEP 1507, 112 (2015) [arXiv:1412.5535].

[26] Kronecker, L., Mathematische Werke IV, 313-318, 1881.

[27] A. Levin and G. Racinet, (2007) math/0703237.

[28] F. Brown and A. Levin, (2011).

[29] B. Enriquez, [arXiv:1301.3042].

[30] J. Fay, Springer 1973.

[31] D. Mumford and M. Nori and P. Norman, Birkhäuser 1983, 1984, 9780817631109, Vol. 2.

[32] J. Broedel, N. Matthes and O. Schlotterer, J. Phys. A 49, no. 15, 155203 (2016) [arXiv:1507.02254].

[33] J. Broedel, N. Matthes and O. Schlotterer, https://tools.aei.mpg.de/emzv.

[34] D. Calaque, B. Enriquez and P. Etingof, Progr. Math. 269 (2009) 165.

[35] R. Hain, [arXiv:1309.0580].

[36] B. Enriquez, Selecta Math. (N.S.) 20 (2014) 491. 